\title{
GMR
}

\section{Effects of prolonged anesthesia with dexmedetomidine, fentanyl, or remifentanil on the self-renewal of mouse embryonic stem cells}

\author{
N. Zhang ${ }^{1}$, Y.R. Cai ${ }^{1}$, X.W. Yi ${ }^{2}$ Y.N. Xiao ${ }^{1}$, B. Chen ${ }^{1}$ and W.X. Li \\ 'Department of Anesthesiology, Eye, Ear, Nose, and Throat Hospital, \\ Fudan University, Shanghai, China \\ 2Department of Anesthesiology, Zhong Shan Hospital, Fudan University, \\ Shanghai, China \\ Corresponding author: W.X. Li \\ E-mail: wenxianli66@gmail.com
}

Genet. Mol. Res. 14 (4): 17809-17819 (2015)

Received August 11, 2015

Accepted October 2, 2015

Published December 22, 2015

DOI http://dx.doi.org/10.4238/2015.December.22.5

ABSTRACT. Previous study has indicated that exposure to anesthesia in early development leads to neuro-apoptosis and is followed by longterm cognitive dysfunction. Given that larger numbers of pregnant women currently receive anesthesia during the first trimester, we wanted to mimic this process in vitro using mouse embryonic stem cells (mESCs) and to explore how different anesthetics affect the self-renewal of mESCs. In the present study, mESCs were exposed to dexmedetomidine, fentanyl, or remifentanil at clinical concentrations for $48 \mathrm{~h}$. The mESCs were then analyzed for cell proliferation and apoptosis. Furthermore, we used flow cytometry to analyze the cell cycle and quantitative real-time polymerase chain reaction to detect the gene expression during the cell cycle as well as the relevant stemness markers. We found that prolonged anesthesia with dexmedetomidine or fentanyl significantly inhibited mESC proliferation, with fewer cell numbers as well as decreased expression of cyclin $B$ and cyclin 
E mRNA compared to that in the control group; meanwhile, $p 21$ and $R B 2$ gene expression was increased. Additionally, increases or decreases in the proportion of cells in the G1 and S phases, respectively, were observed in the dexmedetomidine- and fentanyl-treated groups. These anesthetics also repressed the gene expression of mESC stemness makers such as Oct4 and Sox2. However, remifentanil seemed to have no significant influence on the self-renewal of mESCs. These results demonstrated that prolonged anesthesia with dexmedetomidine or fentanyl, but not remifentanil, inhibited mESC proliferation by blocking the $\mathrm{G} 1$ to $\mathrm{S}$ transition, and repressed the maintenance of $\mathrm{mESC}$ stemness.

Key words: Dexmedetomidine; Remifentanil; Mouse embryonic stem cells; Fentanyl; Self-renewal

\section{INTRODUCTION}

Increasing evidence indicates that exposure to anesthetic and analgesic agents during early development might induce toxicity in the nervous system, resulting in neuro-apoptosis as well as in long-term deficits in learning and memory (Lei et al., 2014). Many intravenous anesthetics commonly used in clinical treatment have been proven to show neurotoxicity. As one of the most popular opioid analgesics for patients undergoing surgery or suffering from chronic pain, fentanyl is commonly used for neonatal anesthesia as well (Kouvaras et al., 2008). A pilot study on prenatal cortical activity showed that fentanyl significantly induced different appearances of spontaneous activity transients (SATs) along with changed electroencephalographs in preterm babies, which might potentially imply the existence of brain damage (Malk et al., 2014). These studies suggested that anesthesia might exhibit toxicity towards brain development. In contrast, many investigators have reported that dexmedetomidine, a potent $\alpha 2$-adrenoreceptor agonist with a high affinity for each of the $\alpha 2$-adrenoceptor subtypes (Peng et al., 2013), did not induce neurotoxicity and cellular degeneration in neither neonatal rodents nor primates (Palanisamy, 2012; Koo et al., 2014). Furthermore, remifentanil, a phenylpiperidine analgesic agent with a high affinity to $\mu$-opioid receptors and exhibiting ultra-short-action, has gradually become established in clinical use (Rogliani et al., 2013), and large studies in vivo have validated its safety in neonates as an analgesic (Demirel et al., 2014).

Almost all anesthetic agents are capable of passing through the placental barrier during a specific gestational period and can occur for most or longer than the time of anesthesia when used for obstetric anesthesia (Lang et al., 2002). Many studies have focused on the anesthetic exposure of pregnant animals during the middle-late trimester to identify potential adverse effects on their offspring. For example, exposure to sevoflurane anesthesia during the middle trimester of pregnant mice has been shown to induce acute neurotoxicity and cognitive impairment in offspring through neuroinflammation, caspase-3 activation, or synaptic loss (Zheng et al., 2013). In addition, previous studies have also shown that infants exposed to maternal regional anesthesia during the late trimester exhibited altered brain structure, including greater local volumes in many functional regions as detected by magnetic resonance imaging (MRI) (Spann et al., 2014). Furthermore, although few studies focused on first trimester anesthesia, it was also found that first trimester anesthesia exposure increased the risks of adverse pregnancy such 
as spontaneous abortion and lower birth weight (Allaert et al., 2007). Additionally, exposure to ketamine/xylazine, isoflurane, buprenorphine, and meloxicam in early pregnancy also reduced fetal mouse growth and the length of the fetal humerus (Thaete et al., 2013). Together, these findings imply that anesthetics have a severe influence on early embryonic development. However, there are few relative studies and the underlying mechanisms of anesthetic toxicity are unknown. We therefore set out to discover how anesthetics influence the nascent embryo during early pregnancy.

More attention has recently been focused on the neurotoxicity of anesthesia on neural precursor cells or neurons; however, less emphasis has been placed on determining the effect of anesthesia on embryonic stem cells (ESCs), which represent the original life form. ESCs are derived from the inner cell mass, which possesses the potential for unlimited proliferation and differentiation into the three germ layers (Yu et al., 2013). Therefore, ESCs are often used as an important in vitro model to explore the early development of embryos (van Dartel et al., 2014). ESCs express the genes for Oct4, Sox2, and Nanog, which maintain their self- renewal (Wang and Guo, 2012). Previous studies have indicated that the ESC stemness maintenance, which ensure not easily differentaiting, was dependent on ESC proliferation to some extent (Ruiz et al., 2011). As they differ considerably from other cell types, ESCs have a very unusual cell cycle structure, most notably characterized by the short length of $\mathrm{G} 1$ phase and the high percentage of cells in S phase (Re et al., 2014). Cyclins are cell cycle proteins that are synthesized and degraded during a specific period. Cyclin $B$, which is expressed in $\mathrm{G} 2$, induces progression to mitosis together with cyclin-dependent kinase 1 (Cdk1). In addition, the level of cyclin $E$ increases around the G1/S transition (Abdelalim, 2013), and the cyclin E-Cdk2 pathway promotes the transition of mouse ESCs (mESCs) from G1 to S phase (Wang et al., 2008). Cdkn1a (also known as p21, encoding $\mathrm{P} 21$ ) is an inhibitor of the cyclin E-Cdk2 complex (Wang et al., 2008). Furthermore, retinoblastoma (RB) family proteins bind and repress the E2F family of transcription factor to restrict the G1/S transition (Conklin et al., 2012). mESCs themselves also express functional receptors associated with anesthetics such as GABA, NMDA, opioid, and adrenergic receptors (Kim et al., 2006; Andäng et al., 2008; Kim et al., 2008; Schwirtlich et al., 2010; Akeju et al., 2014). Therefore, while studies have shown that the proliferation of ESCs was crucial to maintain the stemness of ESCs, few researchers have been concerned with the question of how anesthetics influence the self-renewal of ESCs. It is also unknown whether anesthetics influence the early embryonic development via their actions on cell proliferation and cell-cycle control.

To provide guidance to women in early pregnancy regarding obstetric anesthesia, we therefore determined whether three commonly used and mechanistically distinct anesthetic and analgesic agents - dexmedetomidine, fentanyl, and remifentanil - could affect the self-renewal of mESCs. We found that dexmedetomidine and fentanyl inhibited mESC proliferation by restricting cell cycle progression but not by inducing the apoptosis of mESCs or by influencing the maintenance of stemness. In contrast, remifentanil was proven to be relatively safe. In summary, our study provides the theoretical foundation for choosing proper and safe anesthetics in the clinic.

\section{MATERIAL AND METHODS}

\section{Preparation of mESC culture}

mESCs (46C) were cultured with Dulbecco's modified Eagle's medium (DMEM) (Hyclone, 
Logan, UT, USA) in gelatin-coated plates, supplemented with $15 \%$ fetal bovine serum (FBS) (Gibco, Gaithersburg, MD, USA), 1\% non-essential amino acids (Invitrogen, Life Technologies, Carlsbad, CA, USA), 1\% GIn (Invitrogen), 0.18\% $\beta$-mercaptoethanol (Sigma, St. Louis, MO, USA) and lif (Invitrogen), which maintains the undifferentiated state of mESCs. mESCs were cultured at $37^{\circ} \mathrm{C}$ in a humidified incubator with $95 \%$ air and $5 \% \mathrm{CO}_{2}$.

\section{Pharmacologic reagents}

In vitro, mESCs were placed within an incubator and exposed to clinical concentrations of dexmedetomidine, fentanyl, or remifentanil at $0.4 \mu \mathrm{m}, 10 \mathrm{~nm}$, or $10 \mu \mathrm{m}$, respectively, for $48 \mathrm{~h}$ as previously described (Lin et al., 2009; Peng et al., 2013; Xu et al., 2013). The temperature in the incubator was maintained at $37^{\circ} \mathrm{C}$ with $95 \%$ air and $5 \% \mathrm{CO}_{2}$. After $48 \mathrm{~h}, \mathrm{mESCs}$ were collected for performance of the experiments described below.

\section{MTS assay}

According to the protocols of the CellTiter $96^{\circledR}$ AQueous One Solution Cell Proliferation Assay kit (Promega, Madison, WI, USA), an MTS assay was carried out together with detection of the $490 \mathrm{~nm}$ absorbance value by using a multifunctional microplate reader SpectraMax M5 (Molecular Devices, USA).

\section{Immunofluorescence staining}

BrdU $(50 \mathrm{mg} / \mathrm{mL})$ was added to cultures $1 \mathrm{~h}$ before treatment for immunostaining, and then discarded cultures. mESCs were fixed with $4 \%$ paraformaldehyde for 20 min and washed with phosphate-buffered saline (PBS) 3 times. Next, the cells were permeabilized with $0.2 \%$ Triton $\mathrm{X}-100$ for $7 \mathrm{~min}$ and were then washed again three times with PBS. Subsequently, the cells were incubated with PBS containing 10\% FBS at room temperature for $1 \mathrm{~h}$. The cells were incubated with the primary BrdU antibody (Biowold, USA), and dilution rate was 1:500 at $4^{\circ} \mathrm{C}$ overnight and then were washed 3 times with PBS, followed by incubation with the secondary antibody (Biowold, USA) and the dilution rate was 1:1000 at room temperature for $2 \mathrm{~h}$. Finally, cells were counterstained with HO.33342 (Sigma, USA) and then visualized by microscope (ECLIPSE TE2000-U, Nikon, Japan) with the Microsoft of NIS ELEMENTS.

\section{Cell viability}

To determine cell viability, a single cell suspension in PBS $(0.5 \mathrm{~mL})$ was allowed to react with $0.5 \mathrm{~mL}$ trypan blue solution (0.4\%) (Sigma) for $3 \mathrm{~min}$. The total numbers of viable cells (unstained) and total cells (stained and unstained) were calculated. Cell viability $(\%)=$ viable cells/ total cells $\times 100 \%$.

\section{Cell apoptosis analysis}

Cell apoptosis analysis was performed according to the protocol for the AnnexinV-FITC Cell Apoptosis Detection kit (Keygentec, Taoshan, China). 


\section{Quantitative real-time PCR (qPCR)}

We used TRIzol reagent (Invitrogen) to extract the total RNA from mESCs. The mRNA was reverse-transcribed to cDNA by M-MLV Reverse Transcriptase (TaKaRa, Dalian, China). qPCR amplification was performed for 40 cycles using a SYBR Green qPCR Mix (BioRad, Hercules, CA, USA) by using the Stratagene Mx3000P system. Expression of target genes $\left(2^{-\Delta \Lambda C t}\right)$ was normalized against GAPDH. The primer sequence was as followed: Sox2 PF: 5'-GCGGAGTGGAAACTTTTG TCC-3', PR: 5'-CGGGAAGCGTGTACTTATCCTT-3'; Oct4 PF: 5'-CGGAAGAGAAAGCGAACTAG C-3', 5'-ATTGGCGATGTGAGTGATCTG-3'; Rb2 PF: 5'-AACTTCCCCATGATTAGCGATG-3', PR: 5'-GGTTAGAACACTGAAGGGCATTT-3'; P21 5'-GGATGAAGTTGACGAAATCGTGG-3', 5'-TGTT CACGGGGGCTTTTAGAA-3'; Cyclin B PF: 5'-AGAGCTATCCTCATTGACTGGC-3', PR 5'-AACAT GGCCGTTACACCGAC-3'; Cyclin E PF: 5'-GTGGCTCCGACCTTTCAGTC-3', PR: 5'-CACAGTCT TGTCAATCTTGGCA-3'.

\section{Flow cytometry}

mESCs were collected and fixed in ethanol after 48-h treatment for fluorescence-activated cell sorting (FACS) analysis. PBS (1X) and $0.1 \%$ Nonidet P40 were then added to the cell RNase A for $10 \mathrm{~min}$. Propidium iodide was added to a final concentration of $50 \mu \mathrm{g} / \mathrm{mL}$. Cells were analyzed using a flow cytometer (BD FACSCALIBUR, BD Bioscience, USA). The protocol was referenced by the previous studies (A miR-590 Acvr2a Rad51b Axis Regulates DNA Damage Repair during mESC Proliferation).

\section{Statistical analyses}

All statistical analyses used the Student $t$-test. The significance level for all statistical comparisons was set as follows: ${ }^{*} P<0.05 ;{ }^{* *} \mathrm{P}<0.01$; and ${ }^{* * *} \mathrm{P}<0.001$.

\section{RESULTS}

\section{Dexmedetomidine and fentanyl significantly decreased the size of mESC clones and inhibited the proliferation of mESCs, while remifentanil did not}

We observed that after prolonged ( $48 \mathrm{~h}$ ) anesthesia with dexmedetomidine or fentanyl, mESCs proliferated more slowly and presented as smaller colonies compared to the untreated group. However, the remifentanil group showed no change in colony morphology (Figure 1A). We then counted the cell numbers in each group; the dexmedetomidine and fentanyl groups had significantly fewer cell numbers compared to the control group. There was no significant alteration in the remifentanil group (Figure 1B). Next, we performed the cell proliferation analyses using an MTS assay and BrdU incorporation staining assay. We found that the proliferation of mESCs was significantly inhibited by dexmedetomidine and fentanyl, but not by remifentanil (Figure 1C, D). In order to determine whether the decrease in mESC clones size was induced by dexmedetomidine- or fentanyl-mediated apoptosis, we performed cell viability and apoptosis assays and found that there was no significant difference between all groups (Figure 1E, F). 
A

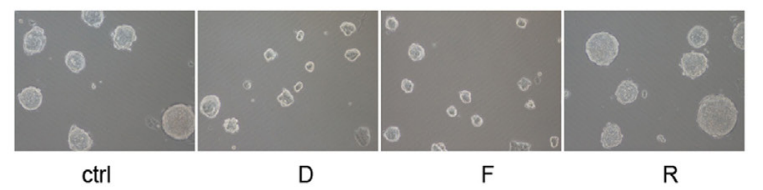

C

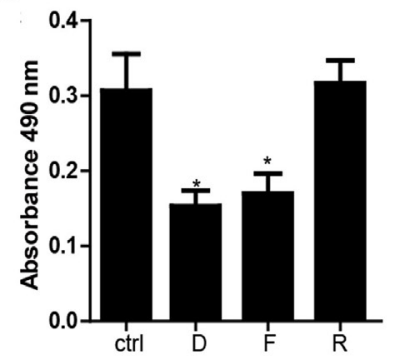

D

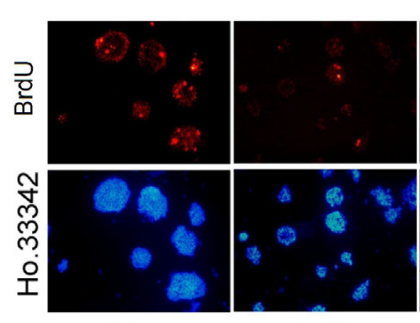

ctrl

D

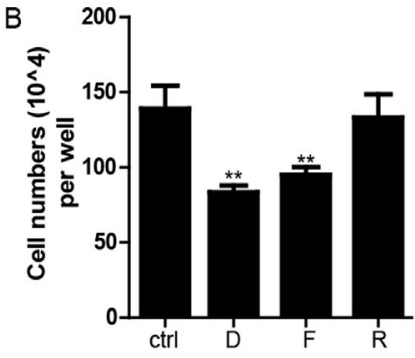

$E$
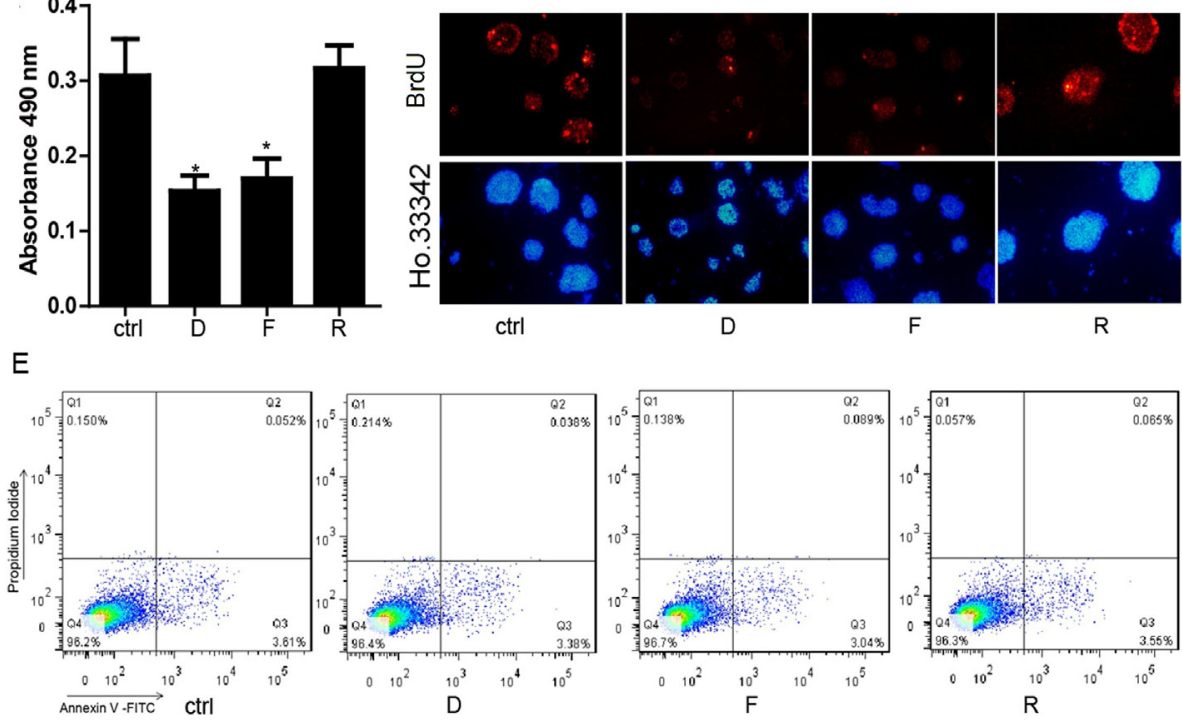

F

$\mathrm{R}$
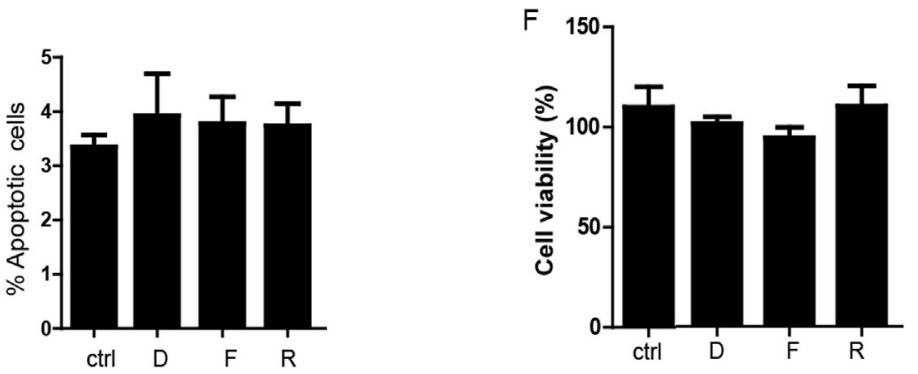

Figure 1. Dexmedetomidine and fentanyl inhibit the proliferation of $\mathrm{mESC}$, while remifentanil does not. A. Colony morphology shows that dexmedetomidine (D) and fentanyl (F) treated groups have smaller colonies of mESCs than do the control (ctr) group. No change can be seen in the remifentanil (R) treatment group. The scale bar represents $100 \mu \mathrm{m}$. B. Dexmedetomidine and fentanyl groups have significantly fewer cell numbers compared to the control group (dexmedetomidine group ${ }^{* *} P<0.01$; fentanyl group ${ }^{* *} P<0.01$ ). The remifentanil group does not influence cell numbers compared to the control group. C. MTS assay and BrdU incorporation staining. D. The dexmedetomidine and fentanyl groups exhibit lower proliferative activities than the control group (dexmedetomidine group, ${ }^{*} \mathrm{P}<0.05$; fentanyl group, ${ }^{*} P<0.05$ ); the remifentanil group does not. E. Cell apoptosis flow cytometric analysis shows that all anesthetic-treated groups are not significantly different than the control group. $\mathbf{F}$. The cell viability assay also shows that no significant difference can be identified between each group. mESCs, murine embryonic stem cells; BrdU, bromodeoxyuridine. Measurement value is represented by measure of the average amount. Error bar represents data of error distribution, The height of error bar is \pm standard error. 


\section{Dexmedetomidine and fentanyl, but not remifentanil, restricted the cell cycle of mESCs and repressed the expression of stemness genes.}

We detected the expression levels of cell cycle related genes and found that the mRNA levels of cyclin Band cyclin $E$ were significantly decreased and the expression level of $p 21$ and $R B 2$ were significantly increased by dexmedetomidine and fentanyl (Figure 2A). FACS analysis of the cell cycle showed that there was a significant increase in the proportion of cells in G1 phase, and a reduction in the proportion of cells in S phase of mESCs after treatment with dexmedetomidine or fentanyl (Figure 2B). We also found that dexmedetomidine and fentanyl down-regulated the expression of Oct4 and Sox2, but not substantially (Figure 2C). In contrast, the remifentanil treatment groups showed no changes in expression of the assayed genes. From the above results, we hypothesized that remifentanil did not affect the proliferation or stemness marker expression of mESCs.
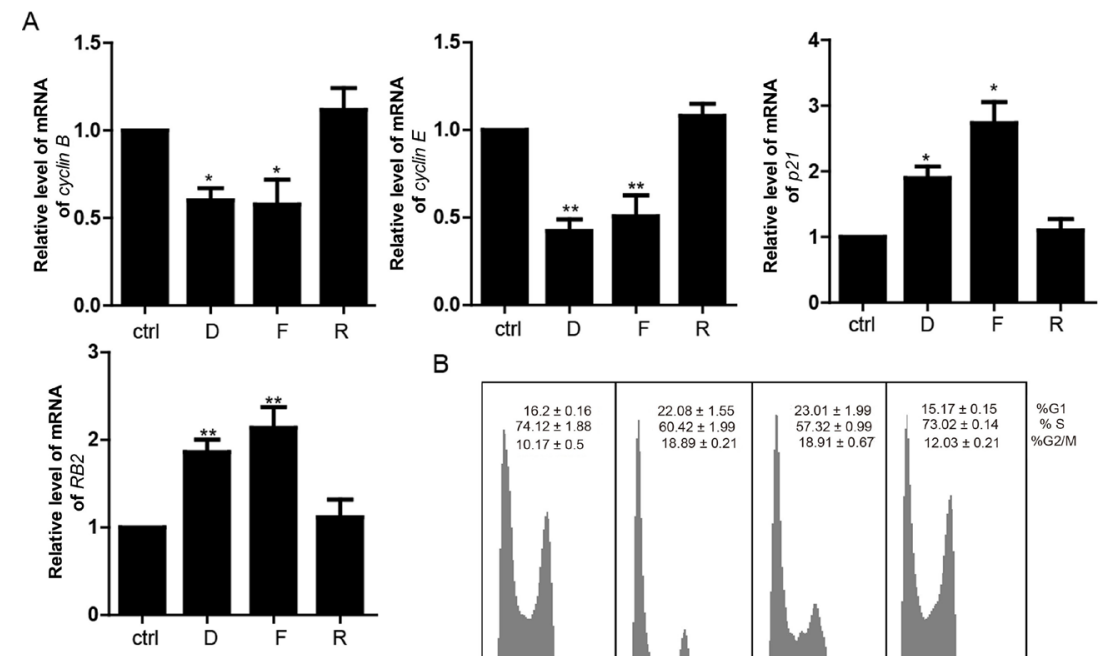

B
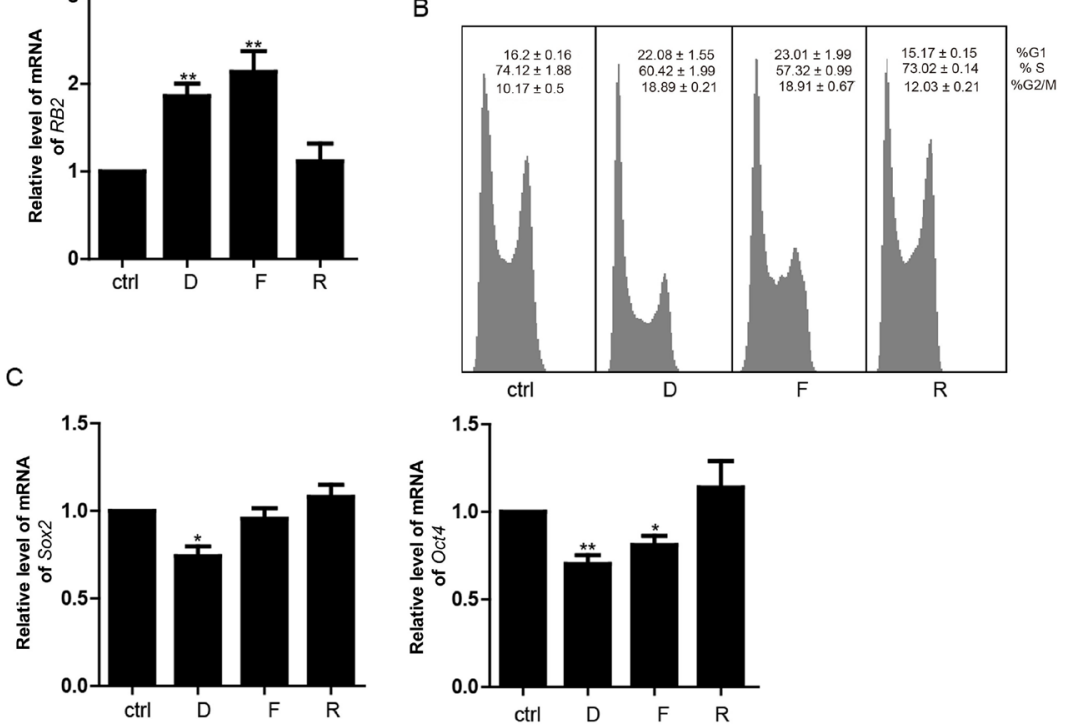

Figure 2. Dexmedetomidine and fentanyl inhibits mESC proliferation via arresting G1-S phase transition and represses the stemness maintenance, while remifentanil does not. A. In the dexmedetomidine and fentanyl groups, the mRNA levels of cyclin $B$ and cyclin $E$ are significantly decreased, and those of $p 21$ and RB2 are significantly increased compared to the control group $\left(\mathrm{N}=10,{ }^{*} \mathrm{P}<0.05,{ }^{*} \mathrm{P}<0.01\right.$, respectively). B. Dexmedetomidine and fentanyl groups exhibit a significant increase in the proportion of mESCs in $\mathrm{G} 1$ phase, and a reduction in the proportion of cells in $S$ phase, while the remifentanil group does not. Data shown are means $\pm S D(N=3)$. C. Detection of the mRNA expression of Oct4 and Sox2 by qRT-PCR ( $N=10,{ }^{*} P<0.05,{ }^{* *} \mathrm{P}<0.01$, respectively). mESCs, murine embryonic stem cells; qRT-PCR, quantitative reverse transcription polymerase chain reaction. 


\section{DISCUSSION}

Anesthesia can do harm to the fetus across the entire pregnancy. In the middle-late trimester, anesthetics primarily exhibit toxicity towards brain development (Zheng et al., 2013; Spann et al., 2014). However, anesthesia poses greater and more serious threats to the mother and child during early pregnancy, including spontaneous abortion and fetal malformation (Allaert et al., 2007; Thaete et al., 2013). Studies on commonly administered maternal anesthetics using in vivo models of rodents have suggested, for example, that exposure to halothane and nitrous oxide during the first trimester could respectively impair learning and weaken the startle reflex reactivity (Palanisamy, 2012). In our study, we wanted to explore the mechanism by which anesthesia might affect the development of embryos during early pregnancy in vitro. Although many studies have examined the functional role of different kinds of anesthetics using various cell types, there have been no previous reports of the ways by which anesthetics influences ESCs. In our study, we used mESCs as a model of early embryonic development, and we chose three commonly used anesthetic and analgesic agents: dexmedetomidine, fentanyl, and remifentanil. From our analyses, we found that prolonged anesthesia with dexmedetomidine or fentanyl significantly inhibited the proliferation of the mESCs, which exhibited fewer cell numbers as compared to the control group, by increasing the population in G1 vs S phase, whereas remifentanil had no significant influence on cell proliferation. However, no significant induction of cell apoptosis was observed for any group. These results might suggest that anesthetic and analgesic agents inhibit the proliferation of mESCs by restricting the cell cycle but not by inducing cell-apoptosis. Next, we tested the expression of genes associated with the cell cycle in mESCs treated with different agents at the mRNA level. Corresponding to the results above, we also found that the expression levels of cyclin $B$ and cyclin $E$ were significantly decreased, whereas the levels of $p 21$ and $R B 2$ were increased in the dexmedetomidine and fentanyl groups. However, the expression of cell cycle genes remained unchanged in the remifentanil group. Finally, we found that the dexmedetomidine and fentanyl groups down-regulated the expression of the mESC stemness genes Oct4 and Sox2, which is comparable to results obtained from previous studies that showed that the inhibition of proliferation can influence the stemness maintenance in ESCs (Ruiz et al., 2011). Considered together, these data pointed to the possibility that dexmedetomidine and fentanyl might inhibit the self-renewal capacity of mESCs by inhibiting cell proliferation and stemness maintenance.

Many lines of evidence have demonstrated that dexmedetomidine could prevent excitotoxic brain injury in neonatal mice (Paris et al., 2006). Furthermore, early studies have shown that dexmedetomidine exerted potent neuroprotection in vivo via the $\alpha 2 \mathrm{~A}$-adrenoceptor subtype in models of neonatal excitotoxic brain damage and hypoxic-ischemic injury (Paris et al., 2006). It has also been confirmed that many adrenoceptor subtypes $(\alpha 1 \mathrm{~A}, \alpha 2 \mathrm{~A}, \beta 1, \beta 2$, and $\beta 3)$ were expressed in mESCs (Kim et al., 2008). Although there has been no data supporting that $\alpha-2$ adrenoceptor was associated with cell cycle control, several reports have indicated that blocking a-1 adrenoceptor yielded G1 or G0/G1 cell cycle arrest in prostate cancer cells (Liou et al., 2009). Nevertheless, we found that exposure to dexmedetomidine at similar or even lower concentrations as those used in previous studies exhibited obvious effects on mESCs including blocking the cell cycle and repressing the maintenance of stemness of mESCs. The underlying mechanisms of dexmedetomidine influencing the self-renewal of mESCs are unknown.

Opioids can activate three classes of opioid receptors ( $\mu \mathrm{ORs}, \delta \mathrm{ORs}$, and $\kappa \mathrm{ORs}$ ) to modulate a variety of biological processes (Anselmi et al., 2013). Many opiates serve as important 
drugs for pain-control. Fentanyl has been shown to exert both pre- and postsynaptic effects via $\mu$-opioid receptors, including decreasing the GABA release at presynaptic terminals in many regions of brain and the GABAergic responses at postsynaptic sites (Griffioen et al., 2004). Additionally, it was found that fentanyl influenced NeuroD in the hippocampus, whose expression is related to neurogenesis and spine stability. This led to a corresponding decrease in miR-190 levels to increase the cellular NeuroD content and thus maintain constant NeuroD levels, and which finally influenced cognitive function (Zheng et al., 2010). Most previous studies have emphasized the impact of fentanyl on the nervous system in vivo; our current experiments have provided additional results indicating that fentanyl inhibited $\mathrm{mESC}$ in vitro in a similar manner as did dexmedetomidine. Furthermore, $\mu$-opioid (MOR-1) and $\kappa$-opioid (KOR-1) receptor expression and immunoreactivity have been described in blastocyst-derived ES cells (Kim et al., 2006). Therefore, perhaps this evidence can provide some clues to explain the mechanisms of the effects of fentanyl on mESCs.

Recent studies have demonstrated that remifentanil preconditioning could exert cardioprotective effects and remifentanil post-conditioning exerted neuroprotective effects by preserving the expression of anti-apoptotic proteins to suppress neuronal cell death in ischemiareperfused heart and cerebral models (Kim et al., 2010; Hu et al., 2013), and that the neuroprotective effect in the hippocampus could attenuate the treatment-associated learning and memory deficits (Hu et al., 2013). In this study, we found that remifentanil had no effect on the self-renewal of $\mathrm{mESCs}$ although it was applied with a longer treatment time than previously utilized; these results are similar to those from previous studies, which demonstrated no significant adverse effects from remifentanil use. It is therefore possible that the nervous system in vivo and mESCs in vitro share similar mechanisms, and that remifentanil can potentially be applied with relative safety in early pregnancy anesthesia.

Currently, there exists a controversial understanding of the relationship between mESC cell cycle progression and the maintenance of stemness. Inhibition of Cdks in mESCs was shown to dramatically block the transition from $G 1$ to $S$ phase with down-regulation of the gene expression of cyclins $B$ and $E$ to inhibit cell proliferation; however, mESCs can still maintain or even increase their Oct4 protein levels (Wang and Guo, 2012). Conversely, other studies had found that a delay in G1-S phase transition in human ESCs (hESCs) could up-regulate dephosphorylated pRb and induce the loss of Oct-4 (Filipczyk et al., 2007). In addition, some studies confirmed that high Oct4 expression increased the inhibitors of the major pRb phosphatase to maintain the hyperphosphorylation of $\mathrm{pRb}$, which influenced the maintenance of stemness and the cell cycle control of stem cells (Schoeftner et al., 2013). Here, we determined that prolonged anesthesia with dexmedetomidine or fentanyl caused rising cell numbers in the G1 phase and down-regulation of Oct4 and Sox 2 gene expression. This might raise the possibility that anesthetics could regulate the cell cycle and the stemness maintenance of mESCs, which in turn contributes to their proliferation and differentiation. However, the mechanisms of these effects remain to be determined.

This study represents the first report to compare different anesthetic and analgesic agents and their effects on the proliferation and stemness maintenance of mESCs. As a next step, we need to verify the effects of anesthetics on hESCs. Furthermore, we should detect the effects of anesthesia applied in vivo in animals during early pregnancy animals to explore the effects on a whole organism and examine the detailed mechanisms underlying these potential effects.

In summary, we provide the first evidence that prolonged $(48 \mathrm{~h})$ anesthesia with dexmedetomidine or fentanyl inhibited $\mathrm{mESC}$ proliferation via blocking the $\mathrm{G} 1$ to $\mathrm{S}$ phase transition, and repressed the maintenance of mESC stemness. However, remifentanil did not exert such 
effects. Our studies suggested that remifentanil might be used with relative safety in anesthesia during early pregnancy. Further study is necessary to clarify the mechanisms of these phenomena and to confirm these results in vivo. Finally, our findings will provide the experimental and theoretical foundations for choosing proper and safe anesthetics for use during early pregnancy.

\title{
Conflicts of interest
}

The authors declare no conflict of interest

\section{ACKNOWLEDGMENTS}

\author{
Research supported by the Laboratory of the Eye, Ear, Nose, and Throat Hospital, Fudan \\ University, China.
}

\section{REFERENCES}

Abdelalim EM (2013). Molecular mechanisms controlling the cell cycle in embryonic stem cells. Stem Cell Rev. 9: 764-773.

Akeju O, Davis-Dusenbery BN, Cassel SH, Ichida JK, et al. (2014). Ketamine exposure in early development impairs specification of the primary germ cell layers. Neurotoxicol. Teratol. 43: 59-68.

Allaert SE, Carlier SP, Weyne LP, Vertommen DJ, et al. (2007). First trimester anesthesia exposure and fetal outcome. A review. Acta Anaesthesiol. Belg. 58: 119-123.

Andäng M, Hjerling-Leffler J, Moliner A, Lundgren TK, et al. (2008). Histone H2AX-dependent GABA(A) receptor regulation of stem cell proliferation. Nature 451: 460-464.

Anselmi L, Jaramillo I, Palacios M, Huynh J, et al. (2013). Ligand-induced mu opioid receptor internalization in enteric neurons following chronic treatment with the opiate fentanyl. J. Neurosci. Res. 91: 854-860.

Conklin JF, Baker J and Sage J (2012). The RB family is required for the self-renewal and survival of human embryonic stem cells. Nat. Commun. 3: 1244.

Demirel N, Bas AY, Kavurt S, Celik IH, et al. (2014). Remifentanil analgesia during laser treatment for retinopathy of prematurity: a practical approach in neonatal intensive care unit. Am. J. Perinatol. 31: 983-986.

Filipczyk AA, Laslett AL, Mummery C and Pera MF (2007). Differentiation is coupled to changes in the cell cycle regulatory apparatus of human embryonic stem cells. Stem Cell Res. 1: 45-60.

Griffioen KJ, Venkatesan P, Huang ZG, Wang X, et al. (2004). Fentanyl inhibits GABAergic neurotransmission to cardiac vagal neurons in the nucleus ambiguus. Brain Res. 1007: 109-115.

Hu X, Xie C, He S, Zhang Y, et al. (2013). Remifentanil post conditioning improves global cerebral ischemia-induced spatial learning and memory deficit in rats via inhibition of neuronal apoptosis through the PI3K signaling pathway. Neurol. Sci. 34: 1955-1962.

Kim E, Clark AL, Kiss A, Hahn JW, et al. (2006). Mu- and kappa-opioids induce the differentiation of embryonic stem cells to neural progenitors. J. Biol. Chem. 281: 33749-33760.

Kim HS, Cho JE, Hong SW, Kim SO, et al. (2010). Remifentanil protects myocardium through activation of anti-apoptotic pathways of survival in ischemia-reperfused rat heart. Physiol. Res. 59: 347-356.

Kim MO, Na SI, Lee MY, Heo JS, et al. (2008). Epinephrine increases DNA synthesis via ERK1/2s through cAMP, Ca(2+)/PKC, and PI3K/Akt signaling pathways in mouse embryonic stem cells. J. Cell. Biochem. 104: 1407-1420.

Koo E, Oshodi T, Meschter C, Ebrahimnejad A, et al. (2014). Neurotoxic effects of dexmedetomidine in fetal cynomolgus monkey brains. J. Toxicol Sci. 39: 251-262.

Kouvaras E, Asprodini EK, Asouchidou I, Vasilaki A, et al. (2008). Fentanyl treatment reduces GABAergic inhibition in the CA1 area of the hippocampus $24 \mathrm{~h}$ after acute exposure to the drug. Neuropharmacology 55: 1172-1182.

Lang C, Behnke H, Wulf H and Geldner G (2002). Placental passage of anesthetics and adjuvants. Anaesthesist 51: $409-417$.

Lei SY, Hache M and Loepke AW (2014). Clinical research into anesthetic neurotoxicity: does anesthesia cause neurological abnormalities in humans? J. Neurosurg. Anesthesiol. 26: 349-357.

Lin H, Higgins P, Loh HH, Law PY, et al. (2009). Bidirectional effects of fentanyl on dendritic spines and AMPA receptors depend upon the internalization of mu opioid receptors. Neuropsychopharmacology 34: 2097-2111.

Liou SF, Lin HH, Liang JC, Chen IJ, et al. (2009). Inhibition of human prostate cancer cells proliferation by a selective alpha1- 
adrenoceptor antagonist labedipinedilol-A involves cell cycle arrest and apoptosis. Toxicology 256: 13-24.

Liu Q, Wang G, Chen Y, Li G, et al. (2014).A miR-590/Acvr2a/Rad51b axis regulates DNA damage repair during mESC proliferation. Stem Cell Reports 3: 1103-1117.

Malk K, Metsäranta M and Vanhatalo S (2014). Drug effects on endogenous brain activity in preterm babies. Brain Dev. 36 : 116-123.

Palanisamy A (2012). Maternal anesthesia and fetal neurodevelopment. Int. J. Obstet. Anesth. 21: 152-162.

Paris A, Mantz J, Tonner PH, Hein L, et al. (2006). The effects of medetomidine on perinatal excitotoxic brain injury are mediated by the alpha2A-adrenoceptor subtype. Anesth. Analg. 102: 456-461.

Peng M, Wang YL, Wang CY and Chen C (2013). Dexmedetomidine attenuates lipopolysaccharide-induced proinflammatory response in primary microglia. J. Surg. Res. 179: e219-225.

Re A, Workman CT, Waldron L, Quattrone A, et al. (2014) Lineage-specific interface proteins match up the cell cycle and differentiation in embryo stem cells. Stem Cell Res. 13: 316-328.

Rogliani P, Calzetta L, Rendina EA, Massullo D, et al. (2013). The influence of propofol, remifentanil and lidocaine on the tone of human bronchial smooth muscle. Pulm. Pharmacol. Ther. 26: 325-331.

Ruiz S, Panopoulos AD, Herrerias A, Bissig K, et al. (2011). A high proliferation rate is required for cell reprogramming and maintenance of human embryonic stem cell identity. Curr. Biol. 21: 45-52.

Schoeftner S, Scarola M, Comisso E, Schneider C, et al. (2013). An Oct4-pRb axis, controlled by MiR-335, integrates stem cell self-renewal and cell cycle control. Stem Cells 31: 717-728.

Schwirtlich M, Emri Z, Antal K, Máté Z, et al.(2013). GABA(A) and GABA(B) receptors of distinct properties affect oppositely the proliferation of mouse embryonic stem cells through synergistic elevation of intracellular $\mathrm{Ca}(2+)$. FASEB J. 24: 1218-1228.

Spann MN, Serino D, Bansal R, Hao X, et al. (2014). Morphological features of the neonatal brain following exposure to regional anesthesia during labor and delivery. Magn. Reson. Imaging. 33: 213-221.

Thaete LG, Levin SI and Dudley AT (2013). Impact of anaesthetics and analgesics on fetal growth in the mouse. Lab. Anim. 47: $175-183$.

van Dartel DA, Schulpen SH, Theunissen PT, Bunschoten A, et al. (2014). Dynamic changes in energy metabolism upon embryonic stem cell differentiation support developmental toxicant identification. Toxicology 324: 76-87.

Wang R and Guo YL (2012). Transient inhibition of cell proliferation does not compromise self-renewal of mouse embryonic stem cells. Exp. Cell Res. 318: 2094-2104.

Wang Y, Baskerville S, Shenoy A, Babiarz JE, et al. (2008). Embryonic stem cell-specific microRNAs regulate the G1-S transition and promote rapid proliferation. Nat. Genet. 40: 1478-1483.

Xu J, Xu P, Li Z, Xiao L, et al. (2013). The role of glycogen synthase kinase-3beta in glioma cell apoptosis induced by remifentanil. Cell. Mol. Biol. Lett. 18: 494-506.

Yu YH, Zhang L, Wu DS, Zhang Z, et al. (2013). MiR-223 regulates human embryonic stem cell differentiation by targeting the IGF-1R/Akt signaling pathway. PLoS One 8: e78769.

Zheng H, Zeng Y, Chu J, Kam AY, et al. (2010). Modulations of NeuroD activity contribute to the differential effects of morphine and fentanyl on dendritic spine stability. J. Neurosci. 30: 8102-8110.

Zheng H, Dong Y, Xu Z, Crosby G, et al. (2013). Sevoflurane anesthesia in pregnant mice induces neurotoxicity in fetal and offspring mice. Anesthesiology 118: 516-526. 